\title{
Optical switching in polymer gels
}

\author{
A. Suzuki, T. Ishii, and Y. Maruyama \\ Department of Materials Science, Yokohama National University, 156 Tokiwadai, Hodogaya-ku, \\ Yokohama 240, Japan
}

(Received 9 August 1995; accepted for publication 27 March 1996)

\begin{abstract}
Soft materials have long been sought after for use in devices such as actuators, artificial muscles, separators, switches, sensors, memories, and so forth. We developed a soft, optically transparent material using polymer gels that can not only be activated by visible light (switched on) but also deactivated (switched off) by altering the local environment using three different means: $p \mathrm{H}$, temperature, and light. This copolymer gel is a covalently cross-linked network of $\mathrm{N}$-isopropylacrylamide, sodium acrylate, and a chromophore, which is found to undergo phase transitions exhibiting large hysteresis in the degree of swelling in response to $p \mathrm{H}$, temperature, and light. In each system, between the transitions for swelling and shrinking, the gel can show either a swollen or a collapsed state, which can be selected according to the history of the variables. It has been established that a thermoresponsive gel with chromophore exhibits a local volume phase transition upon illumination with visible light. By making use of this phenomenon, we have successfully controlled the phase in which a gel exists with visible light: Without light illumination the gel stays in the swollen state. Upon illumination beyond a threshold intensity, however, a volume transition is locally induced, thereby forming a material in which both phases coexist stably for at least several hours after the light source has been removed. The phenomenological stability of the material in the coexistence state is discussed on the basis of the Landau theory. (C) 1996 American Institute of Physics. [S0021-8979(96)07513-5]
\end{abstract}

\section{INTRODUCTION}

A soft material that undergoes changes in shape due to a contractile or expanding force generated in response to chemical environmental changes is a mechanochemical system and its chemical potential energy can be converted into mechanical work. ${ }^{1,2}$ A swelling or shrinking of a gel triggered by changes in the external environment is not specific but rather universal to all gels made of synthetic or natural polymers. Since the discovery of the discontinuous volume phase transition, ${ }^{3}$ the application of gels in devices such as actuators, artificial muscles, controlled delivery systems, sensors, selective pumps, and controlled molecular separators has been suggested. Recently, the volume of a polymer gel has been recognized to result from a balance between the repulsion and attraction of the cross-linked polymer chains within their networks. ${ }^{4}$ The balance arises from a combination of fundamental intermolecular forces, ${ }^{5}$ these include van der Waals force, hydrophobic interaction, hydrogen bonding, and electrostatic force. Control of the forces through the design of polymer networks makes it possible to construct a gel system which undergoes the various types of volume phase transitions $^{6,7}$ in response to a very small and specific difference in external factors, ${ }^{8}$ such as temperature, ${ }^{9}$ light, ${ }^{10,11}$ and stress. $^{12}$

In 1990, the light sensitive $N$-isopropylacrylamide (NIPA) gel, impregnated slightly with the trisodium salt of copper chlorophyllin (chromophore, $\mathrm{CH}$ ) was first synthesized to demonstrate the phase transition due to the local heating of polymer networks upon illumination with visible light. ${ }^{11}$ At equilibrium where the condition of zero osmotic pressure applies, the phenomenological model ${ }^{8,11}$ derived to predict the light-induced phase transition of gels in terms of the gel temperature, $T$ and as a function of the network den- sity, $\phi$ is: $T=T_{\text {gel }}(\phi)$. In accordance with the equation of state of gels based on the Flory-Huggins mean field theory, ${ }^{13}$ this function increases monotonically with $\phi$. It had also been shown previously that the swelling curves of NIPA gels with chlorophyllin molecules are well described by the above equation. ${ }^{8}$ For example, when a light sensitive gel is illuminated by light, the chromophore absorbs the light energy which it subsequently dissipates as heat thereby inducing an increase in the local temperature. The incremental rise of the local temperature is proportional to the incident light intensity as well as to the concentration of $\mathrm{CH}$ that in itself is also dependent upon the network density $\phi$. Thus, it may be assumed that the local temperature of the gel upon light illumination is given by: $T=T_{0}+\alpha I_{0} \phi$, where $T_{0}$ is the ambient temperature, $I_{0}$ is the illuminating light intensity, and $\alpha$ is a constant that is related to the heat capacity of the gel. The relationship between $T_{0}$ and $\phi$ is therefore: $T_{0}=T_{\text {gel }}(\phi)-\alpha I_{0} \phi$. Provided that the light sensitive gel is initially in the vicinity of an unstable region within the temperature-volume phase diagram, the second term on the right-hand side of this equation describes, in general, the ability of local illumination of light to induce a discontinuous gel volume phase transition, ${ }^{11}$ That is, when the light sensitive gel is in thermal equilibrium at a fixed gel temperature $T, \phi$ can discontinuously increase as the intensity of light illumination, $I_{0}$ is increased beyond a threshold value. Conversely, as $I_{0}$ is decreased, $\phi$ decreases discontinuously at almost the same threshold value. In this particular case, the gel does not exhibit hysteresis as a function of $I_{0}$.

Recently, the volume phase behavior of $N$-isopropylacrylamide (NIPA) gel that is randomly but slightly incorporated by both sodium acrylate (SA) and trisodium salt of copper chlorophyllin (chromophore, $\mathrm{CH}$ ) has 


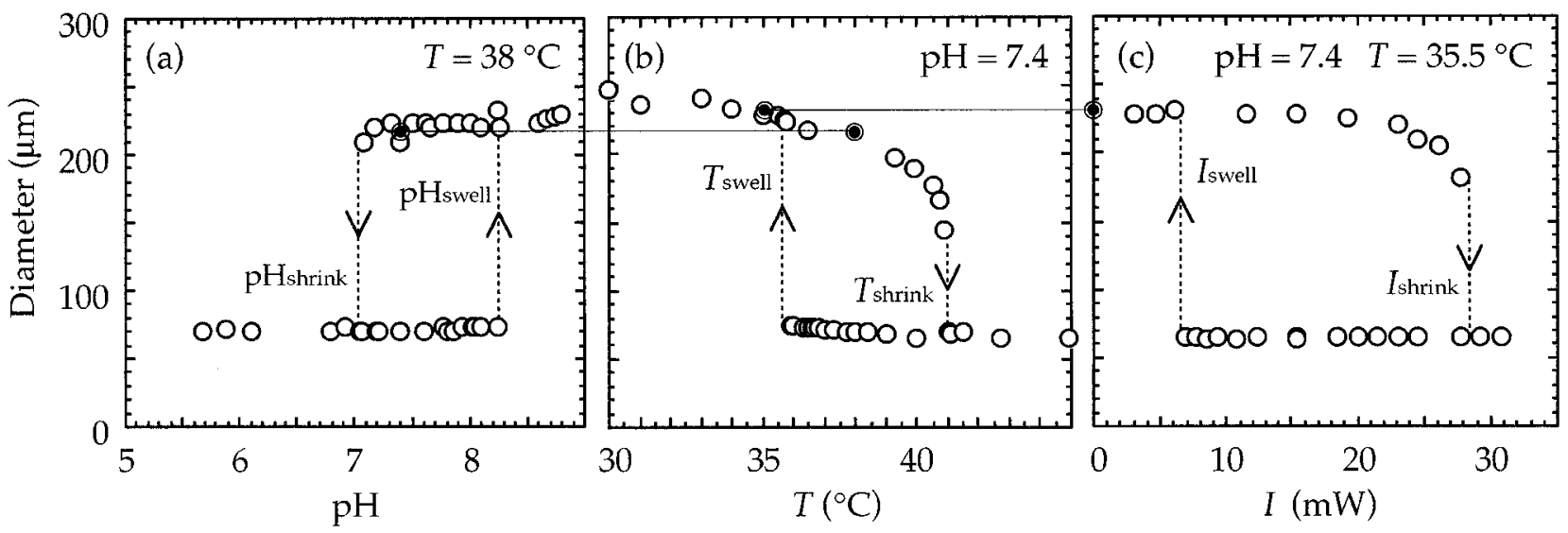

FIG. 1. Equilibrium diameter of the gel as a function of $p \mathrm{H}$ at $38{ }^{\circ} \mathrm{C}(\mathrm{a})$, of temperature, $T$, at $p \mathrm{H}=7.4$ (b), and of visible light intensity, $I$, at $p \mathrm{H}=7.4$ and at $35.5^{\circ} \mathrm{C}(\mathrm{c})$.

been investigated as a function of $p \mathrm{H} \cdot{ }^{14}$ In the present study, it was found that the volume phase transitions of the ionic gel were governed by the extent of hydrophobic interactions or degree of hydrogen bonding induced between the polymers within the gel in response to varying either the temperature/visible light intensity, or $p \mathrm{H}$, respectively. That is, it is now generally accepted that within the ionic gel the $p \mathrm{H}$ of the system mainly affects the degree of hydrogen bonding, whereas the temperature and/or visible light intensity determines the extent of hydrophobic interactions. Independent of either external condition used to induce a volume phase transition, the ionic gel exhibited a large hysteresis upon undergoing a reversible transition between the "shrunken" and "swollen" states. Hysteresis is indicative of a first-order phase transition of the gel and the existence of two free energy minima separated by a free energy barrier. Moreover, at appropriate locations within the volume phase diagram between the swelling and shrinking transitions induced by either external condition, the phases of the gel can coexist. It would, therefore, be possible to control the phase using these phenomena by manipulation of visible light exposure. The purpose of the present article is to describe a gel system developed with a switch function that undergoes a local shrinking transition upon illumination with visible light that is stable even after the light source is removed.

\section{EXPERIMENT}

Gels were designed by several different schemes in which the chemical compositions were varied. We have synthesized several kinds of gels which exhibit large hysteresis in the swelling curves. The gels used in the present experiments were prepared by a free radical copolymerization in water at the ice temperature overnight. ${ }^{11,14}$ A capillary with an inner diameter of $142 \mu \mathrm{m}$ was used to make a uniform cylindrical shape. ${ }^{8}$ The reagents (the amounts in $100 \mathrm{~g}$ of water) were as follows: purified NIPA (7.8 g), SA (94.1 mg), $\mathrm{CH}(72.2 \mathrm{mg}), N, N^{\prime}$-methylenebisacrylamide $(133 \mathrm{mg})$ as cross linker, $N, N, N^{\prime}, N^{\prime}$-tetramethylethylenediamine (240 $\mu \mathrm{l})$ as accelerator, and ammonium persulfate $(400 \mathrm{mg})$ as initiator. The present NIPA gels have slight incorporation of both $\mathrm{SA}$ and $\mathrm{CH}$ monomers, the total number of which is only $3 \%$ of the number of NIPA monomers. After gelation, the gels were taken out and placed in a glass microcapillary with an inner diameter of $1.35 \mathrm{~mm}$. The capillary was encapsulated in a transparent cell, within which water with temperature regulated with an accuracy of $\pm 0.05^{\circ} \mathrm{C}$ was circulated to control the temperature of the gels. ${ }^{8}$ In the glass microcapillary, $p \mathrm{H}$ controlled water can continuously flow from a reservoir where the $p \mathrm{H}$ is decreased by adding $\mathrm{HCl}$ or increased by adding $\mathrm{NaOH}$. To increase (decrease) $p \mathrm{H}$ in acid (base) range, a large amount of water was added to the solvent instead of adding $\mathrm{NaOH}(\mathrm{HCl})$, in order not to increase the ionic strength. To prevent exposure to the $\mathrm{CO}_{2}$ in the air, the solution was kept under $\mathrm{N}_{2}$ gas atmosphere. The image of the gel was monitored and its equilibrium diameter was determined using an optical microscope with an image processor, after the gels reached equilibrium at each temperature and in a solution with and without light illumination. An argon-ion laser emitting light at a wavelength of 488 $\mathrm{nm}$ was used as a light source. The incident beam with a Gaussian profile and width of approximately $5 \mathrm{~mm}$ was focused on the gel with a lens having a focal length of 350 $\mathrm{mm}$; this produced a focused beam of $50 \mu \mathrm{m}$.

\section{RESULTS AND DISCUSSION}

\section{A. Hysteresis between reversible volume phase transitions}

Hysteresis curves were observed in response to each of the three different ways of environment condition changechange of $p \mathrm{H}$, temperature, and light. In the case of $p \mathrm{H}$ change, the phase transition behavior of the present gel has already been established, ${ }^{12}$ and the swelling curves are known to depend strongly on the solvent $p \mathrm{H}$. Figure 1(a) shows the equilibrium diameter as a function of $p \mathrm{H}$ at $38^{\circ} \mathrm{C}$. The phase transition behavior in response to $p \mathrm{H}$ change has been found to display a typical first-order phase transition when $p \mathrm{H}$ is cycled between 5 and 9. It should also be noted that the reversible phase transition between the swollen and shrunken states occurs at two different values of $p \mathrm{H}: p \mathrm{H}_{\text {shrink }}$ and $p \mathrm{H}_{\text {swell }}$. Namely, the gel undergoes a discontinuous phase transition from an initially collapsed state to a swollen 
(a) $\mathrm{T}=38^{\circ} \mathrm{C}$

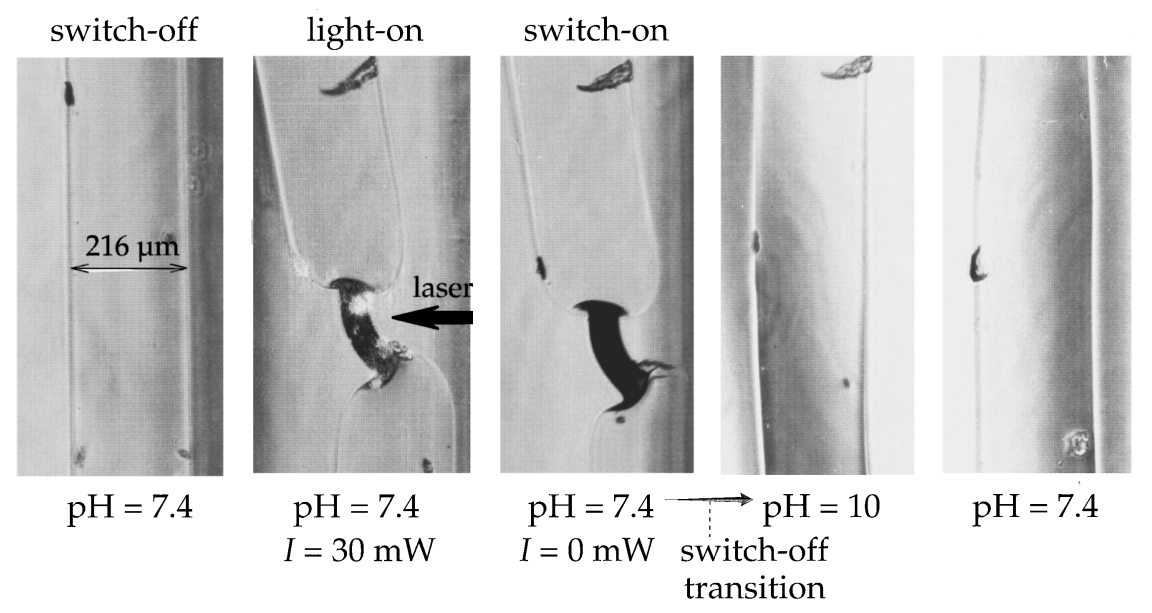

(b) $\mathrm{pH}=7.4$

(c) $\mathrm{pH}=7.4 \quad \mathrm{~T}=35.5^{\circ} \mathrm{C}$
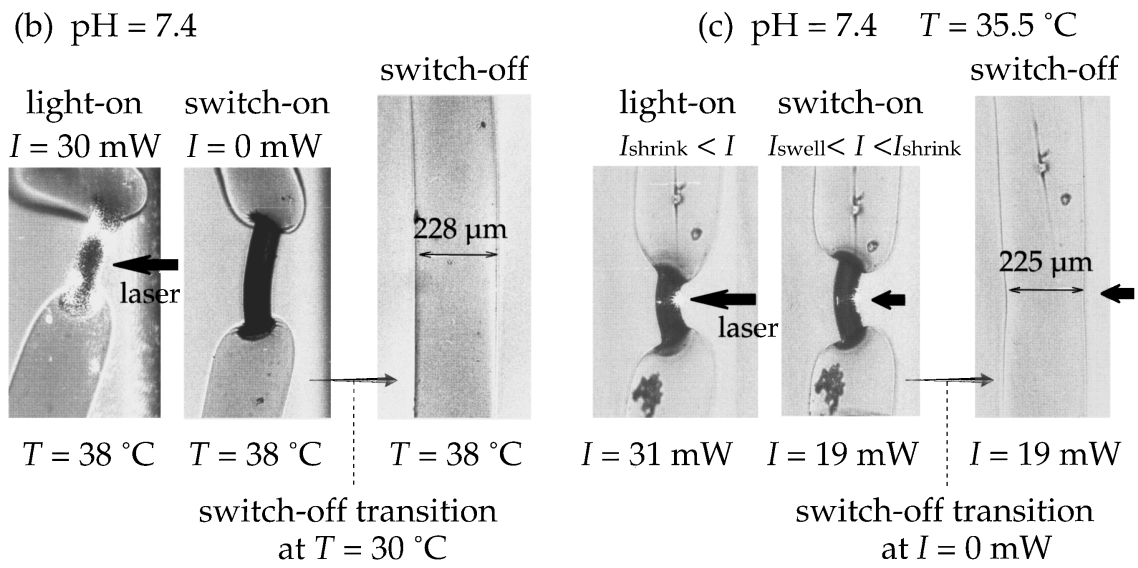

FIG. 2. Photographic sequences showing how the gel can act analogous to a switch that is turned on by visible light, and turned off by $p H$ (a), temperature (b), and the light itself (c); in (a), the initial swollen state (first photograph) at $p \mathrm{H}=7.4$ between the swelling and shrinking transition $p \mathrm{H}$ can enter a coexistence state (second photograph) in response to light illumination from the right to the left-headside, which is stable for several hours after the illumination is removed (third photograph). Swelling occurs at greater $p \mathrm{H}$ than $p \mathrm{H}_{\mathrm{swell}}$ (fourth photograph), and returns to the initial swollen state at $p \mathrm{H}=7.4$ (last photograph). In (b) and (c), the locally collapsed, coexistent (switch on), and the initial swollen state (switch off) are demonstrated in the same order.

state when the $p \mathrm{H}$ is raised to $p \mathrm{H}_{\text {swell }}=8.3$. In order to observe the reverse volume phase transition, the $p \mathrm{H}$ of the system must be lowered well below $p \mathrm{H}_{\text {swell }}$ to $p \mathrm{H}_{\text {shrink }}=7.0$, whereupon the gel returned to the collapsed state. Hence a hysteresis curve was observed as the gel undergoes a reversible phase transition with respect to changes in the solution $p \mathrm{H}$ ranging between $p \mathrm{H}_{\text {swell }}$ and $p \mathrm{H}_{\text {shrink }}$.

The second measurement was conducted by changing the temperature between 30 and $45^{\circ} \mathrm{C}$ at a fixed $p \mathrm{H}$ of 7.4. At the outset, the initial state of the gel, used to determine volume phase transition with respect to temperature change, was the swollen state maintained by a temperature of $38^{\circ} \mathrm{C}$ and $p \mathrm{H}=7.4$. At this temperature and $p \mathrm{H}$ conditions, the initial state of the gel was thus well within the observed $p \mathrm{H}$ hysteresis curve. As the temperature was increased from the starting point of $38^{\circ} \mathrm{C}$, the gel underwent a phase transition from the swollen state to a shrunken state at $T_{\text {shrink }}=41.0^{\circ} \mathrm{C}$. At temperatures beyond $T_{\text {shrink }}$, the collapsed state of the gel was unchanged. To induce the reverse phase transition, the temperature of the system was next lowered. The reverse phase transition from a collapsed state to a swollen state did not, however, occur until the temperature was well below $T_{\text {shrink }}$ at $T_{\text {swell }}=35.8^{\circ} \mathrm{C}$. After repeated cycling of the temperature, the gel exhibited a reversible, reproducible discontinuous phase transition with a large hysteresis loop, as illustrated in Fig. 1(b). As shown, there exist two characteristic temperatures at which the gel underwent a volume phase transition upon heating, $T_{\text {shrink }}=41.0^{\circ} \mathrm{C}$, and cooling $T_{\text {swell }}=35.8^{\circ} \mathrm{C}$. In between these transition temperatures, the phases of the gel can coexist.

In the third experiment, volume changes of the gel were determined as a function of light illumination intensity while maintaining a fixed temperature and $p \mathrm{H}$. In this system, it is necessary to determine the $p \mathrm{H}$ and temperature whereupon illumination with light the gel can locally collapse and subsequently return to its initial state after the illumination is removed. Figure 1(c) shows the relation between diameter and light intensity at a $p \mathrm{H}=7.4$ and $35.5^{\circ} \mathrm{C}$. One can observe a large hysteresis loop in the phase behavior of the gel with respect to light illumination intensity, ranging from the 
shrinking phase transition intensity of $I_{\text {shrink }}=29 \mathrm{~mW}$ to the swelling transition intensity of $I_{\text {swell }}=6.9 \mathrm{~mW}$.

\section{B. On-and-off transition}

To examine the capability of the gels to act analogous to a switch, we performed the following sequence of experiments. (1) The gel was initially maintained at a $p \mathrm{H}=7.4$ and $T=38.0^{\circ} \mathrm{C}$, where it was held only within its swollen state with a diameter of $216 \mu \mathrm{m}$ and well within the $p \mathrm{H}$ hysteresis curve $\left(p \mathrm{H}_{\text {shrink }}<7.4<p \mathrm{H}_{\text {swell }}\right)$. (2) A portion of the gel was then exposed to $30 \mathrm{~mW}$ of laser light for a few minutes, whereupon it was observed that only the illuminated portion of the gel had collapsed to a diameter of $71 \mu \mathrm{m}$. (3) Next, the laser light was removed, but the gel remained in the coexistent state (switch on) for over several hours. (4) Finally, the $p \mathrm{H}$ was raised beyond $p \mathrm{H}_{\text {swell }}$, where the collapsed portion of the gel began to swell and return to its initial swollen state (switch off) at $p \mathrm{H}=7.4$. The entire process is illustrated in Fig. 2(a).

In the second set of experiments, use is made of the hysteresis loop observed in the temperature range between 35.8 and $41{ }^{\circ} \mathrm{C}$ at a fixed $p \mathrm{H}$ of 7.4. Initially, the temperature was fixed at $38{ }^{\circ} \mathrm{C}$, within the range of the hysteresis loop, where the diameter of the gel in the swollen state was 228 $\mu \mathrm{m}$. When the gel was exposed to $30 \mathrm{~mW}$ of laser light for a few minutes (switch on), the local temperature exceeded the swelling transition temperature $T_{\text {swell }}$ and the gel assumed a coexistence state well within the temperature hysteresis loop. The coexistence state was stable for several hours even after the light was removed. When the temperature was lowered below $T_{\text {swell }}$ and brought back to $38^{\circ} \mathrm{C}$, the gel returned to its initial swollen single state (switch off). Shown in Fig. 2(b) is an example of the process.

In the third experimental sequence, the system was not only turned on by visible light, but also turned off by the light itself. In this experiment, the gel was initially in its swollen state (switch off) at $35.5^{\circ} \mathrm{C}$ and $p \mathrm{H}=7.4$. As shown in Fig. 2(c), the gel had collapsed locally when the light intensity was increased beginning from $0 \mathrm{~mW}$ to beyond $I_{\text {shrink }}=31 \mathrm{~mW}$, thus forming a coexistence state (switch on). When the intensity was gradually decreased, the locally collapsed portion of the gel did not achieve its initial swollen state (switch off) at $19 \mathrm{~mW}$. The gel did, however, become swollen when the light intensity was decreased well below $I_{\text {swell }}$ and only after a prolonged period of time.

\section{Stability of coexistence}

We have demonstrated three different ways by which the swollen and collapsed phases of a gel can be made to coexist and remain essentially stable for several hours. That is, the duration of time where both phases coexist upon collapsing locally the gel network with visible light was more than 100 times longer than the characteristic relaxation time normally required by this tiny gel $^{8}(\sim 50 \mathrm{~s})$ to become hydrated from a collapsed state to a swollen state. Thus, it might be important to determine the mechanisms by which the coexistence of both phases can occur over long periods of time, even when the light illumination was removed. For example, the stabil- (a)

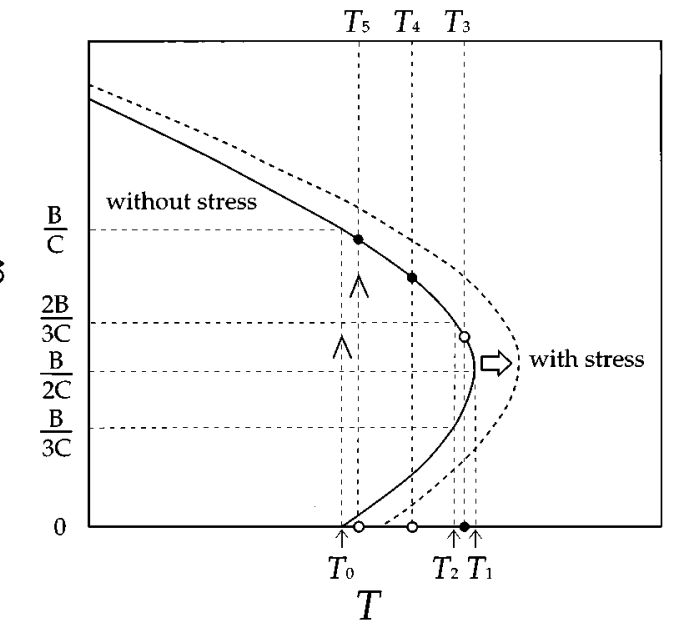

(b)

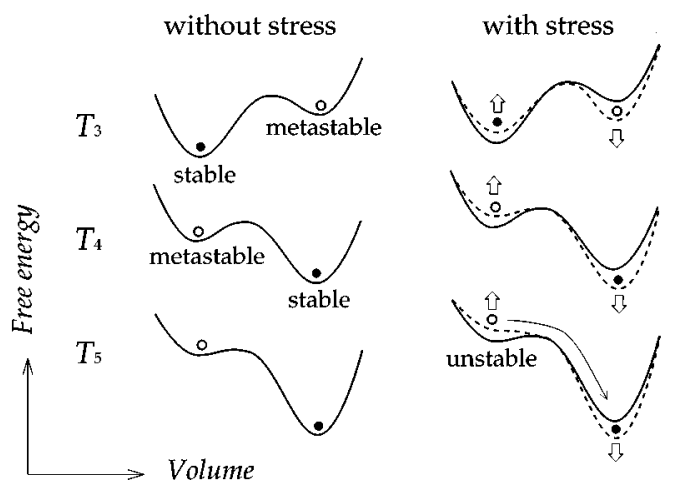

FIG. 3. Change in the order parameter, $\alpha$, as a function of temperature, $T$ (a). Reversible first-order phase transition can be expected with a large hysteresis, while the effect of stretch on the $T-\alpha$ relation can be expressed by the broken curve. Free energy profiles at $T_{3}, T_{4}$ and $T_{5}$ are schematically illustrated in (b). Under small stretch, the coexistence is stable in the local energy minimum at $T_{3}$ and $T_{4}$, while at $T_{5}$ it is unstable and the gel can return to the initial swollen state.

ity of the coexistence phase is expected to be diminished rather than enhanced when the illumination was removed, because the transitional region between the collapsed and swollen phases should be subject to an anisotropic mechanical force that stretches the portion in collapsed state to swell. The problem is in determining whether the locally collapsed state can stay in the local energy minimum under such mechanical constraint. Now, we will discuss the principal mechanism in the case of temperature change by using a phenomenological model based on the Landau theory. ${ }^{14,15}$ In this model the order parameter, $\alpha(0 \leqslant \alpha \leqslant 1)$ can be introduced, which may be related to the physical or chemical quantities in the gel, such as the number of hydrated groups or hydrogen bonding, the osmotic pressure due to counter ions, the repulsive force between ionic groups, and the attractive hydrophobic interaction. If the difference between the repulsive and attractive force could be experimentally obtained, it would probably be a parameter most directly reflecting $\alpha$ in this system. ${ }^{14}$ Then the free energy of the system could be expressed as follows: ${ }^{14}$

$$
F=\frac{A}{2} \alpha^{2}-\frac{B}{3} \alpha^{3}+\frac{C}{4} \alpha^{4},
$$


where $A, B$, and $C$ (defined further below) are positive phenomenological parameters. The cubic term is important in this system, since it is related to the structural symmetry. ${ }^{15}$ According to the Landau theory, only the parameter $A$ depends on temperature as follows:

$$
A=A_{1}\left(T-T_{0}\right),
$$

where $A_{1}$ is a positive constant and $T_{0}$ is the critical temperature. From Eqs. (1) and (2) one can obtain the relation between $\alpha$ and $T$ on the condition that the system prefers the lowest free energy state, which is shown in Fig. 3(a). With increasing $T$, the system remains in the swollen state and enters the metastable swollen state at $T_{2}=T_{0}+2 B^{2} / 9 C A_{1}$, and changes discontinuously to the $\alpha=0$ state at $T_{1}=T_{0}+B^{2} / 4 C A_{1}$. If $T$ is decreased, the system remains in the stable $\alpha=0$ state in $T_{2}<T$ and in the metastable state if $T_{0}<T<T_{2}$. At $T=T_{0}, \alpha$ increases discontinuously, and the system can return to the swollen state. This cycle is a typical first-order phase transition, which corresponds to the phase changes between the collapsed and swollen phases in response to temperature change [Fig. 1(b)]. Between $T_{\text {swell }}$ $\left(=T_{0}\right)$ and $T_{\text {shrink }}\left(=T_{1}\right)$ the gel can enter either of two phases depending on how $\alpha$ is varied prior to that point; the system will choose the one it was in previously. On the other hand, the coexistence observed here is composed of the stable and metastable states, which was realized by local heating of the polymer networks. It should, therefore, be necessary to take into account that the locally collapsed portion is subject to a stress by proximity to the swollen portion, which is maximum at the boundary between the two phases. According to the phase transition in mechanically constrained NIPA gels, it has been established ${ }^{8,12}$ that the stretch of NIPA gel induces the increment of the transition temperature; the collapsed state just above the transition point can return to the swollen state upon application of a small uniaxial stress. Such an effect could be understood in terms of the above theory; the elongation affects the free energy by changing the parameters such as $T_{0}$ (increase for NIPA gels) and also $A_{1}$ since near $\alpha=0$ the square term is dominant in Eq. (1) (note that the magnitude of hysteresis can be expressed as $\left.B^{2} / 4 C A_{1}\right)$. For the particular case of the coexistence phase, the transitional boundary region between the collapsed and swollen phases could also affect the free energy. Hence, the $T-\alpha$ curve should be modified as schematically shown by the broken curves in Fig. 3; the locally collapsed state, if it is not stretched, is stable at $T_{3}$, and metastable at $T_{4}$ and $T_{5}$, while a small stress can make it unstable at $T_{5}$ where it could return to the swollen phase [Fig. 3(b)]. In order to examine the prediction, we conducted the switch on-off experiment at a $p \mathrm{H}$ of 7.4 at $36^{\circ} \mathrm{C}$, that is, just above $T_{\text {swell }}$ [Fig. 1(b)]. As a result, the locally collapsed state returned to a swollen single state within $30 \mathrm{~min}$ as shown in Fig. 4(a). On the other hand, at $38^{\circ} \mathrm{C}$, that is, almost the middle point between $T_{\text {swell }}$ and $T_{\text {shrink }}$, the coexistence persists for at least several hours, as mentioned previously. One can conclude that the locally collapsed state would be stable when the collapsed state remains in the local potential minimum, that is, stable enough not to enter the swollen state as a result of the external stress. It should be noted that there (a) $\mathrm{pH}=7.4 \quad \mathrm{~T}=36{ }^{\circ} \mathrm{C}$

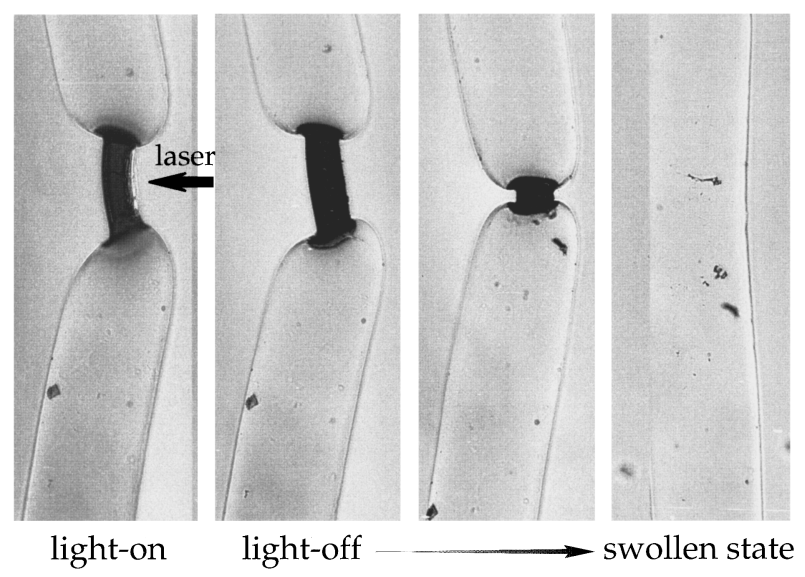

(b)

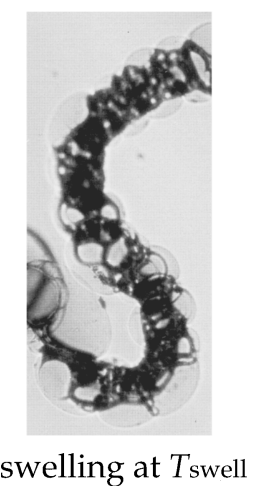

(c)

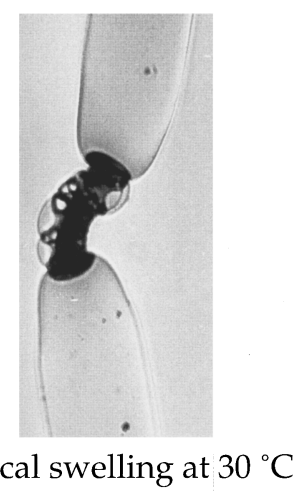

FIG. 4. Photographs of stress-induced phase change where the gel with coexistence at $p \mathrm{H}=7.4$ at $36{ }^{\circ} \mathrm{C}$ just above $T_{\text {swell }}$ can return to the initial swollen phase within $30 \mathrm{~min}$ (a). A bubblelike swelling pattern could always be observed not only at the swelling phase transition but also at the "switchoff' "transition (at $p \mathrm{H}_{\text {swell }}, T_{\text {swell }}$, and $I_{\text {swell }}$ ). Examples are shown during the temperature-related swelling process of the former (b) and the latter (c). Such patterns could not be observed in the stress-induced phase change shown in (a).

appears no bubble like swelling pattern during the stressinduced phase change such as that always observed in each swelling process in response to changes in $p \mathrm{H}$, temperature [Fig. 4(b)], and light intensity. The lack of pattern indicates that the mechanism of the stress induced phase change is essentially different from that of the swelling transition induced by the variables at $p \mathrm{H}_{\text {swell }}, T_{\text {swell }}$, and $I_{\text {well }}$.

\section{Potential use of gel optical switch}

A number of functional polymer gels have been designed and developed for various applications. ${ }^{6}$ Fundamental knowledge of the physics and chemistry of volume phase transition of gels will be of great importance for practical uses. We have demonstrated the switching function of a polymer gel that is controlled by visible light. Visible light is readily available, inexpensive, safe, clean, and easily manipulated to change the gel volume in contrast to other parameters such as solvent composition ${ }^{16}$ and electric field. ${ }^{17}$ It 
can also be delivered in specific amounts with high accuracy. This function of light-sensitive gels is of technological importance in developing various applications not only in the engineering, but also in the biochemical fields. There are a number of parameters that could be varied to make the use of light-sensitive gels as switches practicable. Among these parameters, the on-off switching velocity is essential. In the systems presented here, the phase transition is induced by a local temperature increment that is established as a balance of absorption of light and heat dissipation. Reaching such a stationary state is much faster than the reactions involving ionization and ion migration. ${ }^{10}$ Since thermal diffusion is much faster than collective diffusion of the gel network, the swelling and shrinking process of the gel in response to light is solely governed by the motion of the polymer network. For $1 \mu \mathrm{m}$ diam gels, the response time is expected to be approximately $5 \mathrm{~ms}$, since the time is proportional to the square of diameter. It seems important, however, to solve the problem of the bleaching of the chromophore as well as how to improve the mechanical strength before the system can be used for repeated switching cycles.

The size of a possible switching structure is also an important parameter. Recently, microscopic domain structures have been observed experimentally on NIPA gel surfaces. It had also been evidenced that the magnitude of these structures change during volume phase transitions: ${ }^{18}$ spongelike domains of submicrometer scale were found on the gel surfaces, whose magnitude was strongly affected by the nature of the gel network as well as the environmental conditions, including the condition of mechanical constraint. The reported results disclose that the surface micro-structures of polymer gels in solvent as well as the nanometer scale structural changes are associated with the gel phase transition. One can design a submicrometer switch on the gel surface if domains on that order can be controlled by the visible light; when the illuminated light is focused on a small portion of the gel surface, a domain can shrink to a stable collapsed phase; this is similar to the submillimeter switch presented in this article. In this way, one could store or erase an on or an off, in a specific domain of the gel, which is a kind of "memory" or "display."

Optical switches will also provide an additional opportunity to design new actuators, transducers, controlled release systems, and selective pumps that can be triggered without disturbing the system. For future implications of the present investigations, particular emphasis can be placed on carrying out further the physical basis of the phase behavior under mechanical constraint as well as the dynamic properties of the structural change of gels.

\section{SUMMARY}

We examined the properties of gels with large hysteresis; these are polyelectrolyte gels exhibiting a switching function prepared by incorporating a chromophore into a cross-linked copolymer network consisting of NIPA and SA. This thermoresponsive polymer gel with chromophore is expected to exhibit a local volume phase transition upon illumination with visible light. We found that the gel undergoes volume phase transition in response to changes in $p \mathrm{H}$, temperature, and light intensity, with threshold variables for swelling and shrinking. Between the transitions for swelling and shrinking where the gel can show either swollen or collapsed state, the gel exhibited a local volume phase transition upon illumination with visible light, and the two phases can coexist as metastable states. These confirmed that the gel can have an optical switching effect. The increment of temperature in the immediate vicinity of the polymer chains using visible light is an ideal trigger for induction of a switching function. We believe that the switching phenomena presented here is of technological importance in developing various optical applications such as display units and sensors. We are currently investigating the physical basis of the kinetics, and how to change the environmental conditions as quickly as possible and without great perturbation of the system.

\section{ACKNOWLEDGMENTS}

We express sincere thanks to Dr. S. Gorti for his critical reading of this manuscript. This work was supported in part by a Grant-in Aid from the Ministry of Education, Science and Culture, Japan, and by a Grant of SCF from the Science and Technology Agency, Japan.

${ }^{1}$ W. Kuhn, B. Hargitay, A. Katchalsky, and H. Eisenberg, Nature 165, 514 (1950).

${ }^{2}$ I. Z. Steinberg, A. Oplatka, and A. Katchalsky, Nature 210, 568 (1966).

${ }^{3}$ T. Tanaka, Phys. Rev. Lett. 40, 820 (1978).

${ }^{4}$ T. Tanaka, M. Annaka, F. Ilmain, K. Ishii, E. Kokufuta, A. Suzuki, and M. Tokita, NATO ASI Ser. H64, 683 (1992).

${ }^{5}$ F. Ilmain, T. Tanaka, and E. Kokufuta, Nature 349, 400 (1991).

${ }^{6}$ For recent topics of the gel phase transition, see Adv. Polym. Sci. 109, 110 (1993).

${ }^{7}$ M. Annaka and T. Tanaka, Nature 355, 430 (1992).

${ }^{8}$ A. Suzuki, Adv. Polym. Sci. 110, 199 (1993).

${ }^{9}$ Y. Hirokawa and T. Tanaka, J. Chem. Phys. 81, 6379 (1984).

${ }^{10}$ A. Mamada, T. Tanaka, D. Kungwatchakun, and M. Irie, Macromolecules 23, 1517 (1990)

${ }^{11}$ A. Suzuki and T. Tanaka, Nature 346, 345 (1990).

${ }^{12}$ A. Suzuki and S. Kojima, J. Chem. Phys. 101, 10003 (1994).

${ }^{13}$ P. J. Flory, Principles of Polymer Chemistry (Cornell University Press, Ithaca, NY, 1953)

${ }^{14}$ A. Suzuki and H. Suzuki, J. Chem. Phys. 103, 4706 (1995).

${ }^{15}$ L. D. Landau and E. M. Lifshitz, Statistical Physics (Pergamon, London, 1962).

${ }^{16}$ T. Tanaka, I. Nishio, S.-T. Sun, and S. Ueno-Nishio, Science 218, 467 (1982).

${ }^{17}$ T. Tanaka, D. J. Fillmore, S.-T. Sun, I. Nishio, G. Swislow, and A. Shah, Phys. Rev. Lett. 45, 1636 (1980).

${ }^{18}$ A. Suzuki, M. Yamazaki, and Y. Kobiki, J. Chem. Phys. 104, 1751 (1996) 